\title{
Decidable Reasoning in UML Schemas with Constraints
}

\author{
Anna Queralt and Ernest Teniente \\ Universitat Politècnica de Catalunya \\ \{aqueralt, teniente\}@lsi.upc.edu
}

\begin{abstract}
In this paper we propose an approach to reason on UML schemas with OCL constraints. We provide a set of theorems to determine that a schema does not have any infinite model and then provide a decidable method that, given a schema of this kind, efficiently checks whether it satisfies a set of desirable properties such as schema satisfiability and class or association liveliness.
\end{abstract}

Keywords: Conceptual modeling, Reasoning, Decidability.

\section{Introduction}

A conceptual schema consists of a taxonomy of classes together with their attributes, a taxonomy of associations among classes, and a set of integrity constraints over the state of the domain, which define conditions that each instance of the schema must satisfy. These constraints may have a graphical representation or can be defined by means of a particular general-purpose language.

The Unified Modeling Language (UML) has become a de facto standard in conceptual modeling of information systems. In UML, a conceptual schema is represented by means of a class diagram, with its graphical constraints, together with a set of user-defined constraints, which are usually specified in OCL.

Due to the high expressiveness of the combination of both languages, checking the correctness of a UML conceptual schema manually becomes a very difficult task, specially when the set of textual constraints is large. For this reason, it is desirable to support the designer in reasoning on a conceptual schema.

There are several reasoning tasks that can be performed to determine the correctness of a schema, such as satisfiability of the schema, liveliness of a class or association or reachability of certain states of the information base. Several efforts have already been devoted to reasoning on conceptual schemas. There are automatic procedures for the verification of some properties of schemas in Description Logics [12] or to reason about different kinds of cardinality constraints 3.4.5, but they do not deal with general integrity constraints.

Since the problem of reasoning with integrity constraints in its full generality is undecidable, two different approaches can be followed, either based on decidable procedures for certain restricted kinds of constraints [67, or based on semidecidable procedures for highly expressive constraints 8 . 
In this work we take a mixture of both directions. In particular, our approach consists in translating a UML schema, with OCL constraints satisfying mild syntactical restrictions, into a logic representation to reason on it. Then we provide a set of conditions that guarantee that the schema does not have any infinite model, so that the problem becomes decidable. In this way, any theorem prover or reasoning method can be used knowing that any reasoning task performed on the schema will terminate.

Additionally, once we have determined that all the models of a schema are finite, and since the logic representation of our UML and OCL schemas follows a specific syntactical structure, we provide a reasoning procedure that always terminates and works more efficiently than in the general case.

\section{Base Concepts}

Throughout the paper, $a, b, c, a_{1}, b_{1}, \ldots$ are constants. The symbols $X, Y, Z$, $X_{1}, Y_{1}, \ldots$ denote variables. Sets of constants are denoted by $\bar{a}, \bar{b}, \bar{c}, \overline{a_{1}}, \overline{b_{1}}, \ldots$ and $\bar{X}, \bar{Y}, \bar{Z}, \bar{X}_{1}, \bar{Y}_{1}, \ldots$ denote sets of variables. Predicate symbols are $p, q, r$, $p_{1}, q_{1}, \ldots$ A term is either a variable or a constant. If $p$ is a n-ary predicate and $T_{1}, \ldots, T_{n}$ are terms, then $p\left(T_{1}, \ldots, T_{n}\right)$ is an atom, which can also be written as $p(\bar{T})$ when $n$ is known from the context. An atom is ground if every $T_{i}$ is a constant. An ordinary literal is defined as either an atom or a negated atom, i.e. $\neg p(\bar{T})$. A built-in literal has the form of $A_{1} \omega A_{2}$, where $\mathrm{A}_{1}$ and $\mathrm{A}_{2}$ are terms, and the operator $\omega$ is either $<, \leq,>, \geq,=$ or $\neq$.

A normal clause has the form

$$
A \leftarrow L_{1} \wedge \ldots \wedge L_{m} \text { with } m \geq 0
$$

where $A$ is an atom and each $L_{i}$ is a literal, either ordinary or built-in. All the variables occurring in $A$, as well as in each $L_{i}$, are assumed to be universally quantified over the whole formula. $A$ is often called the head and $L_{1} \wedge \ldots \wedge L_{m}$ is the body of the clause. A set of normal clauses is called a normal program. The definition of a predicate symbol $p$ in a normal program $P$ is the set of all clauses in $P$ that have $p$ in their head. A normal program $P$ is hierarchical if there is a partition $P=P_{1} \cup \ldots \cup P_{n}$ such that the following condition holds for $\mathrm{i}=1,2, \ldots, \mathrm{n}$ : if an atom $r(\bar{T})$ occurs positively or negatively in the body of a clause in $P_{i}$ then the definition of $r$ is contained within $P_{j}$ with $j<i$.

Terms, literals and the syntactic structures made of them are expressions. If $E$ is an expression, then constants $(E)$ and variables $(E)$ are the sets containing the constants and variables, respectively, occurring in $E$.

A substitution $\theta$ is a set of the form $\left\{X_{1} / T_{1}, \ldots, X_{n} / T_{n}\right\}$, where each variable $X_{i}$ is unique and each term $T_{i}$ is different from $X_{i}$. The term $T_{i}$ is called a binding for $X_{i} . \theta$ is called a ground substitution if each $T_{i}$ is a constant.

Let $E$ be an expression and $\theta=\left\{X_{1} / T_{1}, \ldots, X_{n} / T_{n}\right\}$ a substitution. Then $E \theta$ is the expression obtained from $E$ by simultaneously replacing each occurrence of the variable $X_{i}$ in $E$ by the term $T_{i}$.

A fact is a normal clause of the form: $p(\bar{a}) \leftarrow$, where $p(\bar{a})$ is a ground atom. 


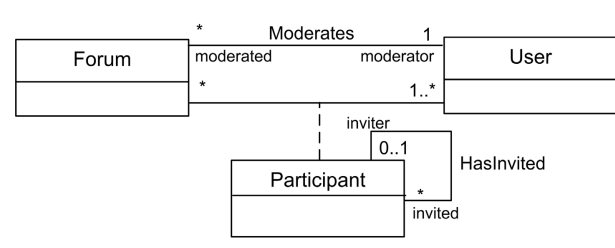

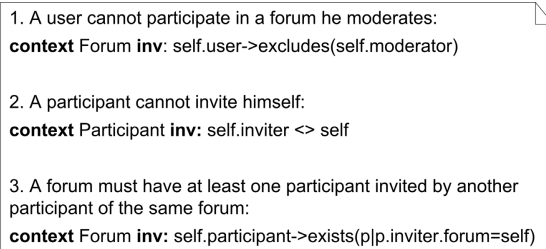

Fig. 1. Conceptual schema for an internet forum

A deductive rule is a normal clause of the form: $p(\bar{T}) \leftarrow L_{1} \wedge \ldots \wedge L_{m}$ with $m \geq 1$ where $p$ is the derived predicate defined by the deductive rule.

A condition is a formula of the (denial) form: $\leftarrow L_{1} \wedge \ldots \wedge L_{m}$ with $m \geq 1$.

A database schema $S$ is a tuple $(D R, I C)$ where $D R$ is a finite set of deductive rules and $I C$ is a finite set of conditions. Literals occurring in the body of deductive rules and conditions in $S$ are either ordinary or built-in. The predicate symbols in ordinary literals range over the extensional database (EDB) predicates, which are the relations that will be stored directly in the database, and the intensional database (IDB) predicates, which are the relations defined by the deductive rules in $D R$. EDB predicates cannot be derived. Conditions in $I C$ define the integrity constraints of the schema $S$.

Deductive rules as well as conditions are required to be safe, that is, every variable occurring in the head or in negated or built-in atoms of their body must also occur in an ordinary positive literal of the same body.

Our logic representation of a schema has a specific structure, since it is obtained from the translation of a UML conceptual schema, with its constraints specified using a subset of the OCL. In particular, the only IDB predicates that appear are those needed to make the conditions safe. Thus, the conditions of the resulting schema are such that their literals correspond to either (positive or negative) EDB predicate symbols or negative IDB predicate symbols. Predicate symbols occurring in ordinary literals in the bodies of deductive rules are positive EDB predicate symbols, i.e. derivation rules do not include derived predicates. To satisfy this restriction, although OCL constraints can include the operations includes, includesAll, notEmpty, exists and one, these specific operations cannot be recursively combined in an OCL expression.

For a schema $S=(D R, I C)$, a state, instance or model $D$ is a tuple $(E, S)$ where $E$ is an EDB, that is, a set of ground facts about EDB predicates. $D R(E)$ denotes the whole set of ground facts about EDB and IDB predicates that are inferred from a database state $D=(E, S)$. $D R(E)$ corresponds to the fixpoint model of $D R \cup E$.

An instance $D$ violates a condition $\leftarrow L_{1} \wedge \ldots \wedge L_{n}$ if there exists a ground substitution $\theta$ such that $D \models\left(L_{1} \wedge \ldots \wedge L_{n}\right) \theta$. In other words, when $L_{1} \theta, \ldots, L_{n} \theta \subseteq D R(E) . D$ is consistent when it violates no condition in $I C$.

We present in this section a simple UML conceptual schema with a set of OCL constraints. We will use this example to illustrate our approach to determine the absence of infinite models. For the sake of simplicity we do not specify any attributes in the classes. 
As can be seen in Fig. 1] each Forum is related to a User that moderates it, and to a set of users that are its Participants, which can be invited by other participants. The textual constraints impose that the moderator of a forum cannot participate in it, and that each forum must have at least one invited participant, which must be invited by some other participant in the same forum.

The translation of the schema into logic results in the following rules, which specify the implicit, graphical and textual constraints that appear in the UML schema. According to our previous work [8], classes are translated into unary predicates, whereas n-ary predicates represent the associations (or association classes). Conditions 1 to 6 correspond to the referential constraints of associations. In this case, since all associations are binary, two such constraints are needed for each of them (one constraint for each association end). Condition 7 specifies that there cannot be two instances of Participant with the same Forum and User, which is an implicit constraint of association classes. Conditions 8 to 11 are the cardinality constraints of associations (the upper and lower bounds of moderator in Moderates, the lower bound of User in the association class Participant, and the upper bound of inviter in HasInvited). Finally, conditions 12 to 14 are the textual constraints of the schema.

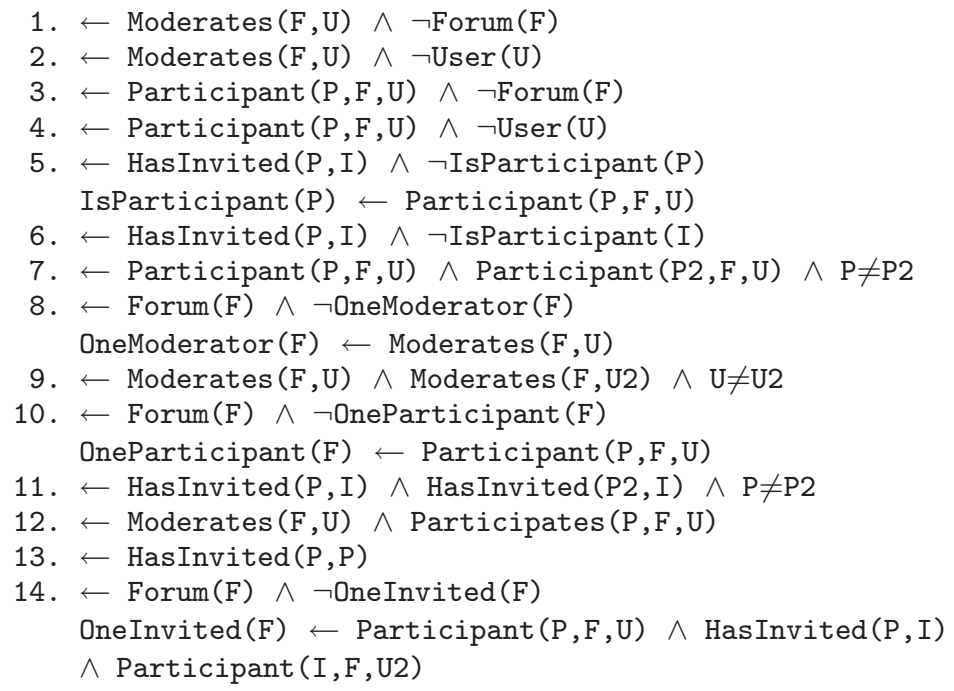

\section{Determining the Decidability of Reasoning on a Schema}

In this section we present our method to identify whether a schema is such that any reasoning task performed on it will terminate, which happens when all its models are finite.

To determine the absence of infinite models, we obtain a graph from the set of constraints of the schema that shows the existing dependencies between them. We formalize the construction of the dependency graph for a given schema in section 3.1, and in section 3.2. we explain how to analyze the graph in order to determine whether all the models of a schema are finite. 


\subsection{The Dependency Graph}

As seen in section 2, a condition consists of a set of positive literals, a set of negative literals and a set of built-in literals. Positive literals are the ones that may violate the constraint, whereas negative literals repair the constraint in case it is violated or, in other words, avoid violation in case that all the positive literals hold in the EDB.

Definition 1. A literal $\mathrm{p}(\bar{X})$ is a potential violation of a condition ic if it appears positively in its body. We denote by $\mathrm{V}(\mathrm{ic})$ the set of potential violations of condition ic.

For example, $V(i c 1)=\{\operatorname{Moderates}(F, U)\}$ since the existence of a fact Moderates $(a, b)$ in the EDB causes the violation of $i c 1$ if the EDB does not contain the fact $\operatorname{Forum}(a)$.

Definition 2. Given a condition ic, there is a repair $\mathrm{R}_{i}(\mathrm{ic})$ for each negative literal $\neg \mathrm{L}_{i}$ in the body of ic. If $\mathrm{L}_{i}$ is base then $\mathrm{R}_{i}(\mathrm{ic})=\left\{\mathrm{L}_{i}\right\}$, otherwise $\mathrm{R}_{i}(\mathrm{ic})$ $=\left\{\mathrm{p}_{1}\left(\bar{X}_{1}\right), \ldots, \mathrm{p}_{n}\left(\bar{X}_{n}\right)\right\}$, where each $\mathrm{p}_{j}\left(\bar{X}_{j}\right)$ is a literal that appears positively in the body of the derivation rule that defines $\mathrm{L}_{i}$.

Each repair of a condition gives an alternative way to avoid its violation. In our example, all conditions have a single repair. For instance, the repair of condition 1 is $R(i c 1)=\{\operatorname{Forum}(F)\}$, and the repair of condition 14 is $R(i c 14)$ $=\{\operatorname{Participant}(P, F, U)$, HasInvited $(P, I)$, Participant $(I, F, U 2)\}$. There are some constraints, such as $i c^{r} \%$, that cannot be repaired once they are violated, unless their potential violations are removed from the EDB.

In the proposed approach, the set $I C$ of constraints of a schema is associated with a directed graph $G$, that we call dependency graph. This graph shows, for each condition $i c_{i}$ of the schema, which conditions may be violated as a result of each possible repair of $i c_{i}$.

Definition 3. A dependency graph $\mathrm{G}$ is a graph such that each vertex corresponds to a condition $\mathrm{ic}_{i}$ of the schema. There is an arc from $\mathrm{ic}_{i}$ to $\mathrm{ic}_{j}$, labeled $\mathrm{R}_{k}\left(\mathrm{ic}_{i}\right)$, if there exists a predicate $\mathrm{p}$ such that $\mathrm{p}(\bar{X}) \in \mathrm{R}_{k}\left(\mathrm{ic}_{i}\right)$ and $\mathrm{p}(\bar{Y}) \in \mathrm{V}\left(\mathrm{ic}_{j}\right)$.

Note that $G$ is sometimes a multigraph, since two different repairs of a condition $i c_{i}$ may lead to the violation of a same other condition $i c_{j}$.

Figure 2 depicts the dependency graph built from the conditions of our example. For the sake of clarity, conditions 5 and 6 have been collapsed in a single vertex, since the predicates belonging to their sets of potential violations and repairs coincide. For instance, it can be seen that the repair of conditions ic 5 and $i c 6$ can violate $i c 4, i c 7, i c 3$ and $i c 12$, since the predicate Participant, which is a repair for conditions $i c 5$ and $i c 6$, belongs to their sets of potential violations.

However, not all the arcs that appear in the graph represent the violation of the condition in the terminal vertex when repairing the initial one. Sometimes, the existence of an arc means the opposite: that the repair of the condition in the initial vertex guarantees the non-violation of the condition in the terminal 


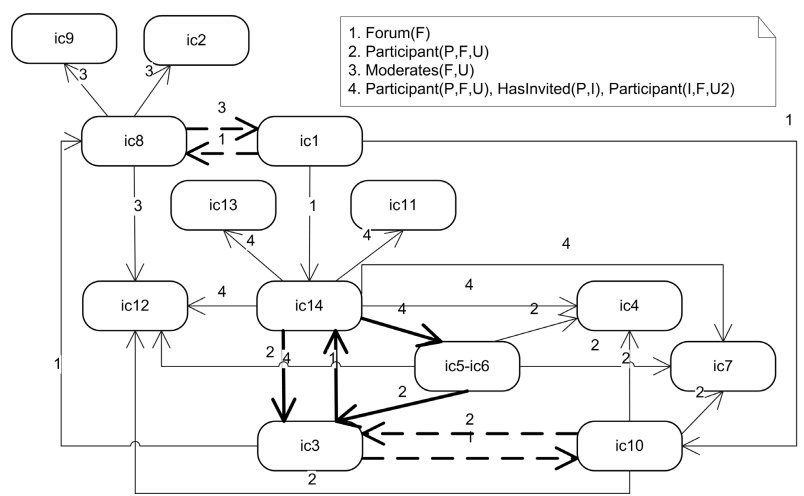

Fig. 2. Dependency graph. Superfluous arcs are dashed, and cycles are highlighted.

vertex. We say this kind of arcs are superfluous. Examples of superfluous arcs, which are depicted with dashed lines in Fig. 2] are the ones between conditions $i c 1$ and $i c 8$. When $i c 1$ is violated due to the insertion of a fact Moderates $(a, b)$ in the EDB, the insertion of the corresponding repair Forum(a) guarantees that $i c 8$ is fulfilled. Similarly, when the first to be violated is $i c 8$ because of the presence of a fact Forum(a), the violation is repaired by the insertion of Moderates $(a, b)$, which guarantees the satisfaction of condition $i c 1$.

Formally, an $\operatorname{arc} r_{i}$ from the constraint $i c_{i}$ to $i c_{j}$ is superfluous when $V\left(i c_{j}\right)=$ $r_{i} \theta$ and there is some repair $R_{k}\left(i c_{j}\right)$ such that $R_{k}\left(i c_{j}\right)=V\left(i c_{i}\right) \theta$, where $\theta$ is a unifier of the sets $V\left(i c_{i}\right) \cup r_{i}$ and $V\left(i c_{j}\right) \cup R_{k}\left(i c_{j}\right)$ that assigns a different term to each distinct variable. This guarantees that $i c_{j}$ is never violated after the repair of $i c_{i}$, since although the facts added by $r_{i}$ potentially violate $i c_{j}$, this condition is always satisfied because its repair also belongs to the EDB. Thus, once these superfluous arcs are identified, they can be left aside since they indicate the ending of any sequence of repairs.

Let $C=\left(\begin{array}{llllll}i c_{1} & r_{1} & \ldots & i c_{n} & r_{n} & i c_{n+1}=i c_{1}\end{array}\right)$ be an alternating sequence of vertices and arcs that define a cycle in a dependency graph $G$. The existence of $C$ implies that the repair $r_{i}$ of a condition $i c_{i}$ may violate other conditions whose repairs could violate $i c_{i}$ again.

As can be seen in Fig. 2, there are two cycles in our dependency graph, defined by the conditions $(i c 3 i c 14)$ and $(i c 3 i c 14 i c 5 / 6)$. Since each constraint has a single repair, an enumeration of vertices suffices to identify each cycle. Note that the existence of superfluous arcs significantly reduces the number of cycles in the dependency graph.

\subsection{Decidability of Reasoning on a Schema}

Our approach to reasoning is aimed at constructing a database state which shows that a certain property holds. That is, a sample EDB where both the particular condition that defines the reasoning task and all the integrity constraints in 
the schema are satisfied. Therefore, our approach requires to perform integrity maintenance when trying to build such a sample EDB.

It can be seen that the constraints that form a cycle in a dependency graph are the only reason for the existence of infinite models. Clearly, a condition that does not belong to a cycle will not cause an infinite sequence of repairs, since it will not be violated again when it has been maintained once for a certain set of facts. On the contrary, constraints that belong to cycles can be violated a potentially infinite number of times, since once they have been maintained, the same facts inserted by the repairs may cause new violations and new repairs, which can result in an infinite model. Then, if we can identify which are the cycles that do not cause an infinite sequence of violations, we can determine whether a schema is suitable to perform any reasoning task in finite time.

In this section we study the cycles of the dependency graph to ensure that the process of integrity maintenance does not loop forever. To do this, we provide a set of theorems that allow to discard the presence of infinite models in the constraints that define each cycle. When all the models of a cycle of constraints are finite we call it a finite cycle.

A first condition that guarantees that a cycle is finite is that it includes a constraint whose violation requires facts that are not inserted in the EDB by some repair in the same cycle. This implies that the cycle will not lead to an infinite sequence of repairs, since there is necessarily a condition in the cycle that will not be violated at some time. This is formalized in theorem 4

Theorem 4. A cycle $C=\left(i c_{1} r_{1} \ldots i c_{n} r_{n} i c_{n+1}=i c_{1}\right)$ is a finite cycle if

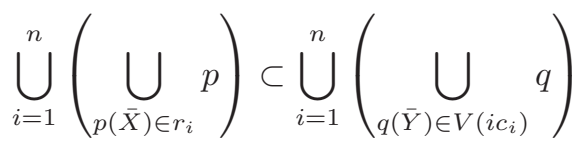

Intuitively, since the union of repairs of the conditions in the cycle is a proper subset of the union of potential violations, at least one potential violation of a constraint $i c_{j}$ in the cycle is an EDB predicate which is not updated during maintenance of the rest of the constraints. Therefore, since the set of facts in the sample EDB at the beginning of the process is finite, $i c_{j}$ may always be violated only a finite number of times.

An example is the following set of constraints, which define a cycle since the repair of the first one is a potential violation of the second, and viceversa:

$\leftarrow \mathrm{p}(\mathrm{X}) \wedge \mathrm{q}(\mathrm{X}) \wedge \neg \mathrm{r}(\mathrm{X})$

$\leftarrow r(X) \wedge \neg \operatorname{aux}(X)$

$\operatorname{aux}(\mathrm{X}) \leftarrow \mathrm{p}(\mathrm{Y}) \wedge \mathrm{Y} \neq \mathrm{X}$

In this example, the potential violation $q(X)$ in the first condition is not added by the repair of the second one. Thus, even when the first constraint is violated because $p(X) \wedge q(X)$ holds in the EDB for some $X$, the repairs of the second condition may only lead to new violations of the first one a finite number of times (one for each fact $q(a)$ contained in the initial EDB when the process of maintaining the previous constraints started). 
A cycle may be finite although it does not satisfy the previous condition. Examples can be found such that all the facts that are potential violations are created inside the cycle and, however, the cycle is not potentially infinite. An example is the cycle (ic3 ic14), which does not satisfy the previous condition but is finitely satisfiable. For instance, when a Participant $(a, b, c)$ is added to the EDB, ic3 requires the insertion of Forum(b) which, in turn, violates ic14. In order to repair this violation, the facts Participant(a2,b,c2), HasInvited(a2,a3) and Participant $(a 3, b, c 3)$ may be inserted, but they will never violate $i c 3$ again.

Definition 5. A variable $\mathrm{x}$ is free in a repair $\mathrm{R}_{i}($ ic $)$ if $\mathrm{x} \in \operatorname{variables}\left(\mathrm{R}_{i}(\mathrm{ic})\right)$ and $\mathrm{x} \notin \operatorname{variables}(\mathrm{V}(\mathrm{ic}))$.

Theorem 6. A cycle $C=\left(\begin{array}{llllll}i c_{1} & r_{1} & \ldots & i c_{n} & r_{n} & i c_{n+1}\end{array}=i c_{1}\right)$ is a finite cycle if $\forall i, 1 \leq i \leq n, \forall p$ such that $\mathrm{p}\left(\mathrm{X}_{1}, \ldots, \mathrm{X}_{m}\right) \in r_{i}$ and $\mathrm{p}\left(\mathrm{Y}_{1}, \ldots, \mathrm{Y}_{m}\right) \in V\left(i c_{i+1}\right)$, $\forall k, 1 \leq k \leq m, \mathrm{X}_{k}$ is free in $\mathrm{r}_{i} \Rightarrow \mathrm{Y}_{k} \notin$ variables $\left(r_{i+1}\right)$.

Intuitively, free variables in a repair are the source of infinity since they propagate the violations to new objects other than the ones that initially violated the constraints in the cycle. The previous condition guarantees that the free variables in the repair of the first constraint are not propagated by the repair of the second constraint. Since such a condition is required for each two consecutive constraints, it is guaranteed that the cycle will not loop forever since no new objects will be infinitely introduced by the repairs.

Applying this condition to the cycle consisting of $i c 3$ and $i c 14$ we can conclude that it is a finite cycle, since the objects added by $i c 14$ (the free variables in its repair) do not appear in the repair of $i c 3$, which means that the new objects are not propagated in the cycle.

There is another cycle in our example, defined by the constraints (ic14ic5/ic6 $i c 3)$, that does not satisfy the previous condition. However, this cycle is not infinite, since the free variables in $i c 14$ are propagated by $i c 5 / i c 6$ but not by $i c 3$, which means that the new objects do not cause a new violation of $i c 14$.

We propose another theorem to identify this kind of cycles, which determines whether all the constraints of a cycle are violated at most once.

Definition 7. Let $\mathrm{C}=\left(\mathrm{ic}_{1} \mathrm{r}_{1} \ldots \mathrm{ic}_{n} \mathrm{r}_{n} \mathrm{ic}_{n+1}=\mathrm{ic}_{1}\right)$ be a cycle in $\mathrm{G}$, where each $\mathrm{r}_{i}$ corresponds to some repair $\mathrm{R}_{j}\left(\mathrm{ic}_{i}\right)$. Let $\mathrm{V}\left(\mathrm{ic}_{i}\right)=\left\{\mathrm{p}_{1}\left(\bar{X}_{1}\right), \ldots, \mathrm{p}_{m}\left(\bar{X}_{m}\right)\right\}$. Then,

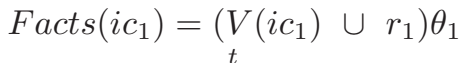

$\operatorname{Facts}\left(i c_{i}\right)=\bigcup_{k=1}^{t} r_{i} \theta_{k} \cup \operatorname{Facts}\left(i c_{i-1}\right), i>1$

where $\theta_{1}$ is a substitution that bounds a distinct constant to each variable, each $\theta_{k}=\theta_{j} \cup \theta_{j}^{\prime}$ is one of the $\mathrm{t}$ possible substitutions such that Facts $\left(i c_{i-1}\right) \models$ $\left(p_{1}\left(\bar{X}_{1}\right) \wedge \ldots \wedge p_{m}\left(\bar{X}_{m}\right)\right) \theta_{j}$ and $\theta_{j}^{\prime}$ assigns a new constant to each variable $X$ such that $X \in \operatorname{variables}\left(\mathrm{r}_{i}\right)$ and $X \notin$ variables $\left(\mathrm{V}\left(\mathrm{ic}_{i}\right)\right)$ if $\theta_{j} \neq \varnothing$, otherwise $\theta_{k}=\varnothing$.

Theorem 8. C is a finite cycle if, for each possible starting $\mathrm{ic}_{i}, \exists k, 1 \leq k \leq n$, such that $\operatorname{Facts}\left(\mathrm{ic}_{k}\right)=$ Facts $\left(\mathrm{ic}_{k+1}\right)$. 
Intuitively, for each $\mathrm{i}>1$, the set Facts $\left(i c_{i}\right)$ extends the set Facts $\left(i c_{i-1}\right)$ by taking into account the repairs required to satisfy $i c_{i}$. Therefore, the condition $\operatorname{Facts}\left(i c_{k}\right)=\operatorname{Facts}\left(i c_{k+1}\right)$ guarantees that the constraint $i c_{k+1}$ is not violated and, hence, the maintenance of the constraints in the cycle will not loop forever.

The previous results allow us to determine whether reasoning on a given conceptual schema will always terminate. Note, however, that this set of theorems is not complete due to the undecidability of this problem.

\section{Reasoning on a Schema}

Once we have determined that all the models of a conceptual schema are finite (as it happens in our example), we can take advantage of the characterization of the logic formulas obtained from our UML and OCL schemas to define a reasoning procedure that works more efficiently than in the general case. Reasoning is concerned with determining the correctness of a schema. Several reasoning tasks have been considered in the literature, such as satisfiability (i.e. checking whether the schema admits a non-empty state that satisfies all the constraints), liveliness of a predicate (i.e. determining whether a certain class or association can have at least one instance) or reachability of partially specified states (i.e. assessing whether certain goals conceived by the designer may be satisfied). In general, each reasoning task can be formulated in terms of a particular goal to attain.

A well-known approach to deal with this problem is to define methods whose purpose is to construct a database state (i.e. an EDB) for which the tested property holds. That is, a sample EDB where both the particular goal to attain and all the integrity constraints in the schema are satisfied. In this way, these methods can uniformly deal with all reasoning tasks.

In this section we propose a new reasoning procedure based on the previous approach. We divide it in two different steps: goal satisfaction and integrity maintenance. These steps are defined in sections 4.1 and 4.2 , respectively.

\subsection{Goal Satisfaction}

Our method is aimed at building a sample EDB which proves that the schema fulfills a specific property defined in terms of a certain goal $G$ to attain. We assume that $G$ is a conjunction of (positive and negative) literals corresponding to EDB predicates and built-in literals; which suffices to handle schema satisfiability, predicate liveliness and reachability of partially specified states [8].

The first step of our method determines the EDB facts that are required to satisfy $G$ without taking into account whether they violate any integrity constraint. Positive literals in $G$ define facts that are necessarily required to satisfy $G$ while negative literals in $G$ identify facts that the sample EDB under construction must not contain. Built-in literals state conditions over the values that the variables of positive and negative literals in $G$ may take.

One of the most difficult tasks is the assignment of concrete values to the variables appearing in $G$ in order to construct the sample EDB. Each possible 
choice defines a different alternative that satisfies $G$, i.e. a different sample EDB. We use Variable Instantiation Patterns (VIPs) 9 for this purpose. These VIPs guarantee that the number of sample EDBs to be considered is kept finite, by taking into account only those variable instantiations that are relevant for the schema, without losing completeness. I.e. the VIPs guarantee that if a solution is not found by instantiating the variables in the goal using only the constants they provide, then no solution exists. VIPs are selected according to the syntactic properties of the schema considered in each test:

1. The Simple VIP: for schemas without negation and integrity constraints.

2. The Negation VIP: for schemas with negation and/or integrity constraints.

3. The Dense Order VIP: for schemas with order comparisons over a dense domain (e.g. real numbers).

4. The Discrete Order VIP: for schemas with order comparisons over a discrete domain (e.g. integer numbers).

In our example, the appropriate VIP is the Negation VIP, since the schema has negation and integrity constraints, but not order comparisons. With this VIP, each variable of the fact to be included in the EDB is instantiated either with a previously used constant or with a new one. For instance, assume that $p(X)$ must be instantiated and that the only constant used up this moment is 0 . Then, according to this VIP, the only relevant instantiations are $p(0)$ and $p(1)$.

Step 1: Goal Satisfaction. Formally, the set $E D B$ of facts required to satisfy $G$ and the set $U n w_{E D B}$ of facts that $E D B$ may never include to fulfill the tested property are obtained as stated in definition 9 . There is a different alternative $E D B$ for each possible substitution $\theta$ provided by the corresponding VIP.

Definition 9. Let $G=\leftarrow P_{1}\left(\bar{X}_{1}\right) \wedge \ldots \wedge P_{n}\left(\bar{X}_{n}\right) \wedge \neg Q_{1}\left(\bar{Y}_{1}\right) \wedge \ldots \wedge \neg Q_{m}\left(\overline{X_{m}}\right) \wedge$ $B_{1} \wedge \ldots \wedge B_{s}$, where $P_{i}, Q_{j}$ are base predicates and $B_{k}$ are built-in literals.

Let $\theta$ be one of the possible ground substitutions obtained via an instantiation of variables $(\mathrm{G})$ and such that $\forall i, 1 \leq i \leq s, B_{i} \theta$ evaluates to true. Then,

- The set of facts required to satisfy $G$ is $\mathrm{EDB}=\left\{\mathrm{P}_{1} \theta, \ldots, \mathrm{P}_{n} \theta\right\}$

- The set of facts unwanted to satisfy $G$ is $\mathrm{Unw}_{E D B}=\left\{\mathrm{Q}_{1} \theta, \ldots, \mathrm{Q}_{m} \theta\right\}$

As an example, assume that the designer wants to check the liveliness of the association Moderates in the conceptual schema of Fig. 1. Moderates will be lively if the goal $G=\leftarrow \operatorname{Moderates}(F, U)$ succeeds for some instantiation. Applying the step 1 of our method we will obtain two different EDBs that satisfy $G$ according to the Negation VIP: $E D B_{1}=\operatorname{Moderates}(0,0)$ and $E D B_{2}=\operatorname{Moderates}(0,1)$.

\subsection{Integrity Maintenance}

Once we have determined the set of EDB facts that satisfies the goal $G$ to attain, the problem of reasoning on the schema may be reduced to that of integrity 
maintenance [10]. Note that, in fact, we already know that the property checked will be satisfied if the EDB resulting from Step 1 does not violate any constraint of the schema. If this is not the case, we must look for additional base facts (i.e. repairs) that make the sample EDB being constructed fulfill all constraints.

Unfortunately, we may not rely on existing integrity maintenance methods to perform this activity. On the one hand, some methods like 1112] can only handle restricted types of integrity constraints which do not cover the kind of constraints we obtain as a result of the translation of the conceptual schema into logic. On the other hand, most methods do not provide an appropriate treatment to the existential variables that appear in the integrity constraint definition 13 14 15 16. The general approach of these methods when instantiating an existential variable is either asking for a value from the user at run-time or assigning an arbitrarily chosen value of the corresponding data type. This is not suitable when using integrity maintenance for reasoning since only a few of the possible alternatives (just one in most cases) would be taken into account to repair a violated constraint. Therefore, this approach does not guarantee the correctness of the result since the impossibility to find a sample EDB would not necessarily imply that the tested property does not hold.

To our knowledge, the most appropriate method to perform the kind of integrity maintenance we require is the CQC-Method [9]. However, and in addition to the decidability drawbacks stated in section [1, the CQC-Method has important efficiency limitations that make questionable its use in practical situations.

Thus, we need to build a new reasoning procedure, which can take advantage both of the dependency graph and the characterization of the logic formulas obtained from our schemas to work efficiently. Since the graph shows the interactions between the constraints, it provides the order in which they should be maintained. In principle, all constraints in the graph must be considered for maintenance since all of them may be violated by the EDB obtained as a result of Step 1. Vertices with no incoming arcs or whose incoming arcs have already been maintained are selected with priority so that a constraint is not considered until all the constraints that may violate it have already been maintained.

An integrity constraint $i c$ must be repaired if its potential violations hold in the sample EDB. Maintenance of $i c$ results in the inclusion of its repairs in the sample EDB being constructed. Note that $i c$ may be violated by several different instantiations of its potential violations. Each of them gives raise to different repairs to be added in the EDB. If a constraint with an empty set of repairs is violated, the sample EDB being constructed must be discarded since it is impossible to make it satisfy such a constraint.

The process of integrity maintenance is formalized as follows. Note that we also use the VIPs to assign concrete values to the existential variables that appear in the repairs of a constraint. Backtracking must be performed each time that the sample EDB under construction reaches a situation where the selected $i c$ can not be repaired. Such backtracking involves considering a different repair of one of the constraints that has been maintained before $i c$. 


\begin{tabular}{|c|c|c|}
\hline & $\begin{array}{c}\text { Selected } \\
\text { constraint(s) }\end{array}$ & Additions to the $E D B$ \\
\hline Step 1: & & $\{$ Moderates $(0,0)\}$ \\
\hline \multirow[t]{3}{*}{ Step 2: } & \begin{tabular}{|l|} 
ic1 \\
\end{tabular} & $\{F$ orum $(0)\}$ \\
\hline & \begin{tabular}{|l|} 
ic10 \\
\end{tabular} & $\{$ Participant $(0,0,1)\}$ \\
\hline & 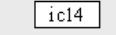 & $\{\operatorname{Has} I n$ ited $(0,1)$, Participant $(1,0,2)\}$ \\
\hline \multicolumn{3}{|c|}{\begin{tabular}{|l|} 
ic13, ic1 1, ic5-6, ic3, ic8 \\
\end{tabular}} \\
\hline & \begin{tabular}{|l|}
$\mathrm{ic} 2$ \\
\end{tabular} & $\{U \operatorname{ser}(0)\}$ \\
\hline \multicolumn{3}{|c|}{\begin{tabular}{|l|} 
ic9, ic7 \\
\end{tabular}} \\
\hline & \begin{tabular}{|l|}
$\mathrm{i} C 4$ \\
\end{tabular} & $\{U \operatorname{ser}(1), U \operatorname{ser}(2)\}$ \\
\hline \multicolumn{3}{|c|}{\begin{tabular}{|l|} 
ic12 \\
\end{tabular}} \\
\hline Sample & & 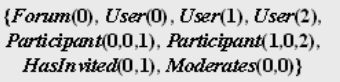 \\
\hline
\end{tabular}

Fig. 3. A sample EDB that proves that Moderates is lively

Step 2: Integrity Maintenance. Let $i c=\leftarrow P_{1}\left(\bar{X}_{1}\right) \wedge \ldots \wedge P_{n}\left(\bar{X}_{n}\right) \wedge$ $\neg Q_{1}\left(\bar{Y}_{1}\right) \wedge \ldots \wedge \neg Q_{m}\left(\bar{X}_{m}\right) \wedge B_{1} \wedge \ldots \wedge B_{s}$ be the condition selected for maintenance from the dependency graph, where $P_{i}, Q_{j}$ are base predicates and $B_{k}$ are built-in literals. Let $E D B_{i}$ be the set of required facts at that moment. Let $\operatorname{Eval} V(i c)$ and $\operatorname{EvalR}\left(R_{i}(i c)\right)$ be the set of built-in literals that appear in the body of $i c$ and in the body of the rule from which $R_{i}(i c)$ is obtained, respectively. Then $E D B_{i+1}$ is computed as follows:

if $R_{i}(i c)=\oslash$ and $\mathrm{EDB}_{i} \models\left(P_{1}\left(\bar{X}_{1}\right) \wedge \ldots \wedge P_{n}\left(\bar{X}_{n}\right) \wedge B_{1} \wedge \ldots \wedge B_{s}\right) \theta_{j}$

then error $(i c$ cannot be repaired)

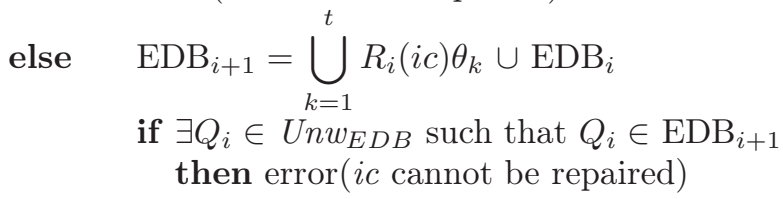

where each $\theta_{k}=\theta_{j} \cup \theta_{j}^{\prime}$ is a substitution such that $\operatorname{EDB}_{i}=(V(i c) \wedge$ $\operatorname{Eval} V(i c)) \theta_{j}$ and $\theta_{j}^{\prime}$ is one of the possible substitutions obtained from an instantiation of all the variables in variables $\left(R_{i}(i c)\right) \backslash \operatorname{variables}(V(i c))$ such that $\operatorname{Eval} R\left(R_{i}(i c)\right) \theta_{j}^{\prime}$ evaluates to true, if $\theta_{j} \neq \oslash$, otherwise $\theta_{k}=\oslash$.

Figure 3 shows an execution of the integrity maintenance step of our method for one of the EDBs obtained in Step 1. Each row in the figure shows the integrity constraint being maintained (as selected through the order defined by the dependency graph) and the additions to the EDB required to repair the constraint, if any. A row contains several constraints when none of them is violated 
by the EDB under construction. As a result of the execution, our method obtains a sample EDB which confirms that the association Moderates is lively.

The constraint $i c 1$ is selected first since it is the only vertex with no incoming arcs in the graph. It is violated since $\mathrm{V}(i c 1)=\operatorname{Moderates}(\mathrm{F}, \mathrm{U})$ holds in the EDB with substitution $\theta_{j}=\{\mathrm{F} / 0, \mathrm{U} / 0\}$. Then, since $\mathrm{R}(i c 1)=$ Forum $(\mathrm{F})$, the repair Forum(0) is added to the sample EDB to ensure that it does not violate $i c 1$.

The next constraint to be selected is $i c 10$ since all its predecessors have already been maintained. Similarly, it is violated since $\mathrm{V}($ ic10 $)=$ Forum $(\mathrm{F})$ holds in the EDB with substitution $\theta_{j}=\{\mathrm{F} / 0\}$. Since $R($ ic 10$)=\operatorname{Participant}(\mathrm{P}, \mathrm{F}, \mathrm{U})$, Participant $(0,0,1)$ is added to the sample EDB since we assume that the substitution obtained is $\theta_{j}^{\prime}=\{\mathrm{P} / 0, \mathrm{U} / 1\}$.

The method proceeds then with $i c 14$ which requires considering two additional repairs whose concrete values have also been obtained via the application of a VIP. The rest of the constraints are either not violated or require repairs which are obtained in the same way than the repairs of $i c 1$.

At the end, the method succeeds and it obtains the sample EDB $=\left\{F_{O}\right.$ rum(0), User(0), User(1), User(2), Participant(0,0,1), Participant(1,0,2), HasInvited $(0,1)$, Moderates $(0,0)\}$. Note that seven additional facts have been added to the EDB to ensure that it does not violate any integrity constraint.

We do not show in this figure the unsuccessful repairs that may have happened during the execution. For instance, when determining repairs for ic10, Participant $(0,0,0)$ could have been considered. However, this alternative does not lead to a valid solution since it violates $i c 12$, which can not be repaired.

\section{Related Work}

In this section we review how reasoning on conceptual schemas has been addressed in the literature. As will be seen, the main contribution of our approach is to deal with more expressive conceptual schemas than previous methods.

The problem of determining the satisfiability of a schema has been widely studied in ER schemas, mostly regarding strong satisfiability of cardinality constraints. This notion was introduced in [5], where the problem was reduced to solving a linear inequality system. In [17] the problem is solved by means of a graph theoretic approach, which was extended in [3] to deal with a generalization of the concept of cardinality. In [4, satisfiability is checked by building a minimal sample database satisfying a set of global cardinality constraints.

More expressive schemas are considered in [67], where the finite satisfiability of object-oriented database schemas is determined. The schemas can be annotated only with specific textual constraints to restrict the value of an attribute by comparing it to another value.

The Alloy language and analyzer [18] provide interesting reasoning capabilities for more expressive schemas by searching for examples of the tested properties. However, since the search space must be limited by the user, failure to find an example does not necessarily mean that one does not exist.

The problem of checking satisfiability has also been addressed for UML conceptual schemas. Restricted UML schemas are analyzed in [19, detecting conflicts 
regarding disjointness and covering constraints in hierarchies, and inconsistencies in the redefinition of inherited cardinality constraints.

A different approach to reason on UML schemas is to translate them into Description Logics (DL) and perform several reasoning tasks using a DL-based system. This allows not only checking the satisfiability of the complete schema but also determining other properties such as class consistency, class equivalence or class subsumption [1, or checking whether a class is forced to have either zero or infinite objects [2]. However, OCL constraints, as well as other UML constructs such as association classes or n-ary associations, are disallowed in order to guarantee decidability.

An alternative approach is not to restrict the expressiveness of the schema and consider general constraints, but then reasoning becomes semidecidable. This direction has been followed in [8], where several reasoning tasks on a UML schema with textual OCL constraints are performed using the CQC-Method as a reasoning engine. In addition to the decidability drawback of this approach, and as far as efficiency is concerned, the reasoning procedure proposed in this paper represents an important improvement regarding the number of integrity constraints that are considered for maintenance.

\section{Conclusions}

We have proposed an approach to reason on UML schemas with OCL constraints. Our approach can deal with almost all the operators that can be used to define an OCL expression (all the boolean operators defined in the OCL standard, as well as select and size, that return a collection and an integer). Exceptions are those expressions resulting from recursively combining includes, includesAll, notEmpty, exists and one. Then, given a conceptual schema of this kind, our method allows determining whether it satisfies certain desirable properties such as schema satisfiability, predicate liveliness or reachability of partially specified states.

Our approach consists of two different tasks, which are the main contributions of our work. First, we analyze whether the schema is such that any reasoning task performed on it will terminate. This is achieved by means of the construction of the dependency graph of constraints and the definition of a set of conditions over this graph that ensure that the schema does not have any infinite model, which are the reason for undecidability.

Second, we define a procedure that allows to efficiently check whether a certain property holds by constructing a sample EDB in which the property is satisfied. Moreover, the impossibility of finding any solution implies that the property does not hold. This procedure is decomposed in two different steps: satisfying the goal that defines the tested property and maintaining all the integrity constraints of the schema to ensure that the sample EDB built is consistent.

As further work, we plan to implement the approach defined in this paper and apply it to practical situations. We would also like to extend our results to minimize the restrictions on the OCL expressions we can deal with. 
Acknowledgements. This work has been partly supported by the Ministerio de Ciencia y Tecnología under projects TIN2005-06053 and TIN2005-05406.

\section{References}

1. Berardi, D., Calvanese, D., de Giacomo, G.: Reasoning on uml class diagrams. Artificial Intelligence 168(1-2), 70-118 (2005)

2. Cadoli, M., Calvanese, D., Giacomo, G.D., Mancini, T.: Finite model reasoning on uml class diagrams via constraint programming. In: AI*IA 2007: Artificial Intelligence and Human-Oriented Computing, pp. 36-47 (2007)

3. Hartmann, S.: On the Consistency of Int-cardinality Constraints. In: Ling, T.W., Ram, S., Li Lee, M. (eds.) ER 1998. LNCS, vol. 1507, pp. 150-163. Springer, Heidelberg (1998)

4. Engel, K., Hartmann, S.: Minimal Sample Databases for Global Cardinality Constraints. In: Eiter, T., Schewe, K.-D. (eds.) FoIKS 2002. LNCS, vol. 2284, pp. 268-288. Springer, Heidelberg (2002)

5. Lenzerini, M., Nobili, P.: On the satisfiability of dependency constraints in entityrelationship schemata. Inf. Syst. 15(4), 453-461 (1990)

6. Formica, A.: Finite satisfiability of integrity constraints in object-oriented database schemas. IEEE Trans. on Knowledge and Data Eng. 14(1), 123-139 (2002)

7. Formica, A.: Satisfiability of object-oriented database constraints with set and bag attributes. Information Systems 28(3), 213-224 (2003)

8. Queralt, A., Teniente, E.: Reasoning on UML Class Diagrams with OCL Constraints. In: Embley, D.W., Olivé, A., Ram, S. (eds.) ER 2006. LNCS, vol. 4215, pp. 497-512. Springer, Heidelberg (2006)

9. Farre, C., Teniente, E., Urpí, T.: Checking query containment with the cqc method. Data and Knowledge Engineering 53(2), 163-223 (2005)

10. Moerkotte, G., Lockemann, P.C.: Reactive consistency control in deductive databases. ACM Trans. Database Syst. 16(4), 670-702 (1991)

11. Console, L., Sapino, M.L., Dupré, D.T.: The role of abduction in database view updating. J. Intell. Inf. Syst. 4(3), 261-280 (1995)

12. Lobo, J., Trajcevski, G.: Minimal and consistent evolution in knowledge bases. J. Applied Non-Classical Logics 7(1-2), 117-146 (1997)

13. Ceri, S., Fraternali, P., Paraboschi, S., Tanca, L.: Automatic generation of production rules for integrity maintenance. ACM Trans. DB Syst. 19(3), 367-422 (1994)

14. Decker, H.: An extension of sld by abduction and integrity maintenance for view updating in deductive databases. In: JICSLP, pp. 157-169 (1996)

15. Schewe, K.D., Thalheim, B.: Towards a theory of consistency enforcement. Acta Inf. 36(2), 97-141 (1999)

16. Mayol, E., Teniente, E.: Consistency preserving updates in deductive databases. Data Knowl. Eng. 47(1), 61-103 (2003)

17. Thalheim, B.: Entity-Relationship Modeling: Foundations of Database Technology. Springer, New York (2000)

18. MIT Software Design Group: The Alloy Analyzer, http://alloy.mit.edu

19. Kaneiwa, K., Satoh, K.: Consistency Checking Algorithms for Restricted UML Class Diagrams. In: Dix, J., Hegner, S.J. (eds.) FoIKS 2006. LNCS, vol. 3861, pp. 219-239. Springer, Heidelberg (2006) 